\title{
ПЕРСПЕКТИВЫ КАЧЕСТВЕННОГО ПРОИЗВОДСТВА РОВНИЦЫ НА РОВНИЧНЫХ МАШИНАХ
}

Казаков Ф.Ф.

Доцент кафедры "Технология и дизайн текстильная

" Бухарского инженерно - технологического института.

Саттарова H.H.

Ассистент кафедры "Технология и дизайн текстильная"

Бухарского инженерно - технологического института.

Ширинова Н.И.

Магистр кафедры "Технология и дизайн текстильная" Бухарского инженерно-технологического института.

\section{PROSPECTS FOR HIGH-QUALITY PRODUCTION OF ROVING MACHINES ON ROVING MACHINES}

\author{
F.F. Kazakov \\ PhD associate professor of the Department \\ "Textile Technology and Design \\ "Bukhara Engineering and Technological Institute. \\ N.N. Sattarov \\ Assistant of the Department \\ "Textile Technology and Design" \\ Bukhara Institute of Engineering and Technology \\ N. I. Shirinova \\ Master of the Department \\ "Textile Technology and Design" of the \\ Bukhara Institute of Engineering and Technology
}

\begin{abstract}
Аннотация. В данном статье дано информация как можно определить интенсивность кручения ленты на ленточном машине. И ещё какие процессы должны провести чтобы непосредственно из такой ленты получить пряжу на кольцевых прядильных машинах. Кроме того о принцип работе на современной ленточной машине $\mathrm{F}$ 15.

Abstract. This article provides information on how to determine the torsion intensity of the tape on the tape machine. And what other processes should be carried out in order to get yarn directly from such a tape on ring spinning machines. In addition, the principle of operation on a modern tape machine $\mathrm{F} 15$.

Ключевые слова: Ленточная машина, волокно, прядильная машина, вытяжной прибор, катушка, ровница, толщина, вытяжка.

Key words: Belt machine, fiber, spinning machine, exhaust device, coil, roving, thickness, exhaust.

Сегодня текстильная промышленность Узбекистана является одной из ведущих и динамично развивающихся отраслей.

В результате практической работы по диверсификации текстильной промышленности страны и стимулированию экспорта готовой продукции потенциал нашей страны в этой области растет.

Лента, полученная на ленточной машине последнего перехода, обладает всеми свойствами, необходимыми для получения из нее пряжи: волокна очищены от посторонних примесей, перемешаны, распрямлены и расположены параллельно оси ленты, выровненной по толщине. Чтобы непосредственно из такой ленты получить пряжу на кольцевых прядильных машинах, ее необходимо утонить в вытяжных приборах высокой вытяжки. Однако в этом случае усложняется конструкция вытяжного прибора, затрудняется обслуживание прядильной машины. Поэтому при кольцевом способе формирования пряжи необходимое утонение ленты осуществляют чаще всего в два этапа [1].

Интенсивность кручения является важной технологической характеристикой, которая определяет прочность продукта, производительность труда ровничниц и производительность машин. Интенсивность кручения можно определить, используя угол наклона волокон к оси продукта, или крутку $K$ (число кручений, приходящихся на 1 м продукта), или коэффициент крутки. Крутка, соответствующая максимальной прочности продукта, называется критической, а соответствующий ей коэффициент крутки называется критическим коэффициентом крутки.
\end{abstract}


Крутку выбирают такой, чтобы ее прочность была достаточной для наматывания на катушку и чтобы ровницу можно было вытягивать в вытяжном приборе с малым усилием без повреждения волокон. Ровница в качестве питающего продукта для кольцевых прядильных машин по сравнению с лентой обладает рядом преимуществ: катушка с ровницей является компактной паковкой, удобной для транспортировки; наличие крутки обеспечивает хорошие условия сматывания ровницы без изменения ее свойств. В зависимости от различных режимов функционирования машины, различаются следующие световые комбинации лампочек, расположенных на машине.

“Крутки”: здесь устанавливаются крутки для производимой ровницы; они выражаются в кр/д (Кручение на дюйм), и при помощи внутреннего подсчёта на этой же строке показывается значение тех же самых круток в кр/м (К. на метр).

Если установить низкую величину круток, может появиться надпись “очень низкие крутки” обозначает, что цилиндровому двигателю была задана скорость выше максимальной; чтобы поддерживать желаемое количество круток необходимо снизить рабочую скорость рогулек (точнее, достаточно понизить самую высокую из скоростей “Начальная скор.”, “Скор. 2”, “Скор.

3” и “Скор. 4”, установленных на этой же самой странице). В случае установки недопустимого значения на машину в рабочем положении, новое значение не будет учитываться, и машина остановится [2].

“Скорость каретки”: Скорость каретки, которая варьируется во время формирования катушек в соответствии с изменениями скорости веретен, выражается в витках на каждый мм патрона: значение должно быть между 0,2 и 0,8. Уменьшая значение витки/мм, возрастает скорость каретки; если это значение слишком низкое, появится надпись “Скорость каретки слишком высокая”; в этом случае, как и в параметре “Крутки”, чтобы поддерживать желаемое значение витки/мм, необходимо снизить рабочую скорость рогулек. В случае установки недопустимого значения на машину в рабочем положении, новое значение не будет учитываться, и машина остановится. Нужно регулировать этот параметр, если витки неслишком большом или слишком маленьком расстоянии друг от друга.

“Предусматриваемое конечное увеличение": этот параметр устанавливается в соответствии с предыдущим, если показатель не изменяется автоматически в фазе инициализации машины, не вводится никакое изменение. Алгоритм расчета регулирует снижение скорости веретен с их поступательным движением вперед, формируя на их конечной части, конечное увеличение.

Когда QCV (виртуальное значение кулачка) достигнет значения 5000, появится мигающая надпись “ изменение линейного увеличения приведено в действие”, и остается в действии до завершения операции. Как правило, на конечной части бобины, выполняется более «мягкая» намотка, для того чтобы компенсировать эффект натяжения, в следствии прогрессивного увеличения диаметра.

“Коррекция натяжения": отношения скорости между рогульками, цилиндром и веретенами подсчитываются программой, согласно установленным условиям и количеству слоёв, намотанных на катушку. Поскольку эти отношения могут меняться в зависимости от скорости вращения механизмов, представляется возможным установить коррекцию для каждой технологической операции: начальная минимальная скорость, средняя скорость, первый слой после закрепления ровницы, скорость импульсов.

Установленная величина, равная 1,000, означает нулевую коррекцию; установив более низкую величину, веретена будут вращаться медленнее: например, установив 0,950, фактическая скорость будет равна теоретической, умноженной на 0,950 (равнозначно 95\%) [3].

В зоне 1 машина начинает (в соответствии с начальным диаметром этой зоны, или “Диаметром 1” обработку при 1300 об/м (с установленной начальной скоростью этой зоны, или “Скоростью 1”) и уменьшает скорость при каждом реверсировании, пока не дойдёт до 1250 об/м (начальная скорость следующей зоны, или “Скорость 2”) в соответствии с диаметром катушки, равным 80мм (начальный диаметр следующей зоны, или “Диаметр 3”);

Таким же образом, в следующих зонах машина продолжает последовательно изменять скорость (обновляемую с каждым реверсированием каретки) так, чтобы в начале каждой зоны работать с начальной скоростью этой зоны, и прийти к концу этой зоны со скоростью, равной начальной скорости следующей зоны.

Как и в предыдущих примерах, последняя зона (здесь зона 4) представляет собой исключение; поскольку у неё нет последующей зоны, конечная скорость равна начальной этой же самой зоны: это означает, что скорость всей конечной зоны неизменна. Зона 1 Зона 2 Зона 3 Зона 4 (конечная).

В зависимости от различных режимов функционирования машины, различаются следующие световые комбинации лампочек, расположенных на машине. Для изменения установок, касающихся управления, необходимо ввести соответствующий код доступа “контроль датчиков”: контроль правильного функционирования датчиков производства SQ31, вращения рогулек SQ32, вращения веретен SQ38 “контроль дистанционных выключателей”: для выявления какой-либо непоследовательности, которая могла бы указать на неисправность дистанционных выключателей (например, КМ1 активирован в то время, как КМ0 ещё выключен).

“Контроль давления”: контролирует наличие сжатого воздуха; может быть лучше исключить этот контроль в случае ремонта обменника (единственное устройство, использующее сжатый воздух); 
“центрирование ремней веретен”: эта команда позволяет прокрутить только веретена, чтобы отрегулировать у них центрирование приводных ремней;

“центрирование ремней рогулек”: эта команда позволяет прокрутить только рогульки, чтобы отрегулировать у них центрирование приводных ремней;

A - Настройки группы приводов Siemens $611 \mathrm{U}$

В начале формирования присутствуют следующие операции:

Минимальная скорость способствует закреплению ровницы на пустом патроне в установленное время (скорость и время устанавливаются с клавиатуры: рекомендуется 250 об/м за 4 сек.).

Средняя скорость способствует натяжению ровниц и перекрытию конца закрепления: такая скорость (устанавливаемая с клавиатуры: рекомендуется скорость в 500 об/м) остаётся включенной пока каретка не достигнет величины AQ6.

После AQ6 машина достигает рабочей скорости.

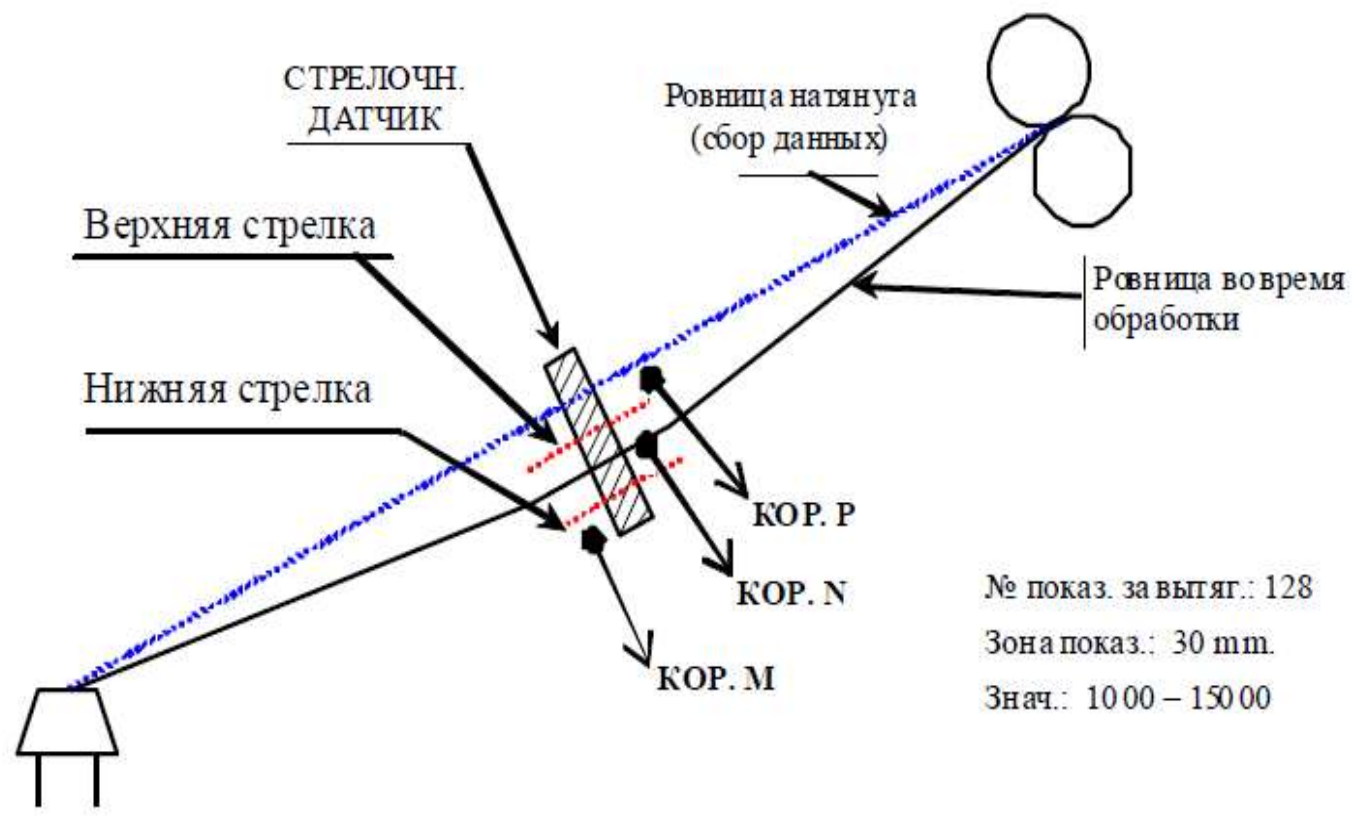

В зоне между цилиндром и входом рогулек, как правило, расположенны ${ }^{\circ} 2$ стрелочных датчика (CCD1 и CCD2), направленные на определение положения соответствующих ровниц во время формирования; отмеченное положение, описанное числом между 1`000 и 15`000, является средним из 128 показанный, снятых в промежутке между двумя последующими реверсированиями каретки; полученное таким способом число передаётся на ПЛК, который его учтёт при внесении последующих корректировок (временных) в значение прироста “виртуального кулачка".

Положение ровниц снимается во время всего пробега кроме зон “исключения остановки” перед реверсированием [4].

Показатели сопоставляются ПЛК с установленными ограничениями значений (см. далее) и на основании этого сопоставления выбираются последующие корректировки, чтобы применить во время следующего вытягивания. 


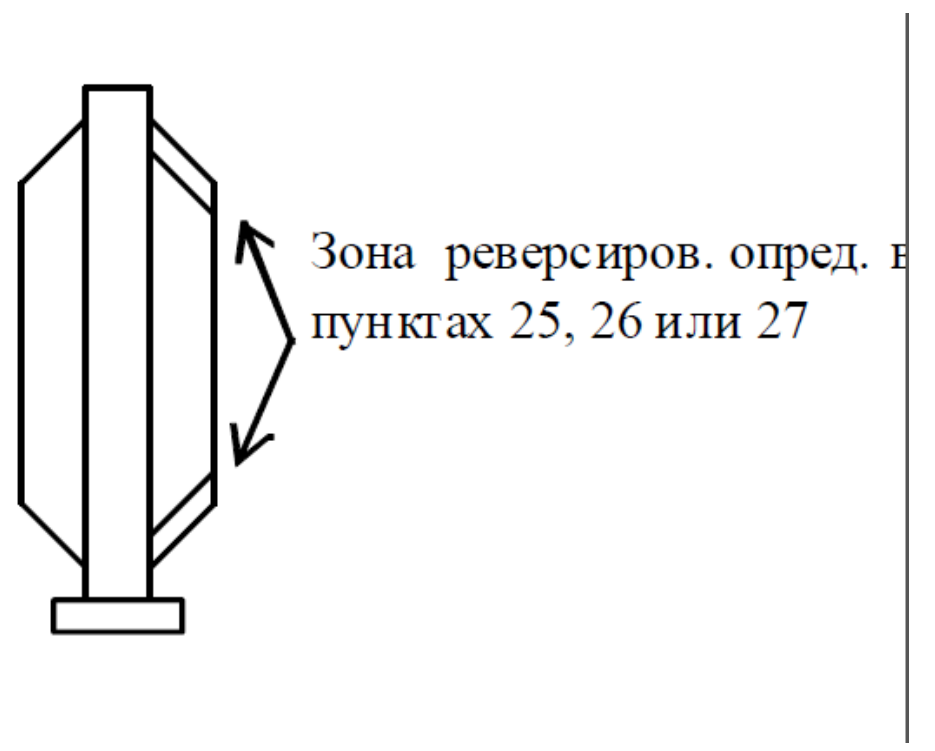

Включение стрелочных датчиков

1. Для правильной настройки стрелочных датчиков целесообразно выключить и включить напряжение в машине, это позволит Плате CP-340 ПЛК и Плате ROJ правильно синхронизироваться, после чего нужно действовать следующим образом:

2. Введите код доступа УРОВ. 3 “ввод данных”;

3. Подключите стрелочные датчики клавишей ВКЛЮЧЕНЫ

4. Посредством клавиши выведите на дисплей Стр. Дисп. 67 “Настройки ROJ - 2";

5. - клавишей ON подключите датчики CCD1 и CCD2

6. посредством клавиши СБОР ДАННЫХ CCD1 включите сбор данных датчика CCD1

7. вручную отрегулируйте натяжение ровницы, соответствующей датчику CCD1, и одновременно нажмите клавишу для сохранения значения в памяти: на дисплее можно будет прочитать мгновенное значение, которое будет соответствовать значению натянутой ровницы.

8. Повторите операции 6, 7, 8 для датчика CCD2. Причины остановки могут быть следующие:

а. Ручное управление

Нажимается одна из красных кнопок ОСТАНОВКИ/STOР, расположенных на машине или Р50 на клавиатуре;

b. Обрыв ленты или ровницы

В этом случае остановка невозможна в зоне реверсирования катушки и происходит уже после реверсирования, чтобы избежать падения витка катушки (рис. 12-а).

\section{с. Автоматическое вмешательство}

Конец формирования, перебег каретки, защита от перегрева и т.д.

ПРИМЕЧАНИЕ: зона исключения остановки из-за обрыва ленты или ровницы должна регулироваться в соответствии со скоростью каретки:

- вызовите на дисплей Стр. Дисп. 25, 26 или 27 (в зависимости от выбранного типа завязки);

- с помощью стрелок перейдите на строку, соответствующую “исключению остановки”;

- измените величину (выраженную в мм) и подтвердите выбор посредством ENTER/BВОД;

Исключ.

остановки

останов

зона

ПРИМЕЧАНИЕ: можно работать даже только с одним включенным датчиком. Определение рабочей зоны При каждой команде нормальной остановки все двигатели синхронно останавливаются в установленное программой время замедления (обычно 6").

При каждой команде аварийной остановки или отсутствии электрического напряжения все двигатели остаются синхронизированными между собой и подпитываются самими же двигателями, которые при торможении действуют как генераторы электроэнергии; время остановки зависит каждый раз от состояния машины (скорости разных механизмов и количества уже намотанной на катушки ровницы) [5]. 
Всегда на Стр. Дисп. 67, переместите курсор в конец страницы дисплея на строчки, соответствующие предельным размерам, и установите значения: рекомендуемые значения для верхних пределов - 200 и для нижних пределов- 400.

Нужно иметь ввиду, что при увеличении этих значений, происходит ослабление ровниц. Автоматически определяются следующие поля:

"КОР. N" - зона колебания ровницы, подвергающейся оптимальному натяжению намотки, поэтому прирост следующего вытягивания будет равным Номинальному; “КОР. Р”-зона колебания слишком натянутой ровницы, поэтому прирост следующего вытягивания увеличится (Номинальный + Коррекция) “COR. P”- “KOP. M” - зона колебания слишком ослабленной ровницы, поэтому прирост следующего вытягивания уменьшится (Номинальный - Коррекция) “COR.

На стр. Дисп. 44 “Отображение на дисплее устройства ROJ” можно одновременно увидеть значения, снятые стрелочными датчиками, и прирост, подсчитанный ПЛК.

В случае если установленные операции не выполняются в ожидаемое время или заблокированные, машина подаёт аварийный сигнал, и на дисплее клавиатуры отображается соответствующее сообщение.

Операции конца формирования и повторного запуска машины; любая операция должна завершиться в течении 60 секунд, в противном случае, включается аварийная сигнализация;

\section{Порядок анализа и коррекции}

Контроль двух веретен вместо одного позволяет уменьшить количество ошибок в расчётах, возникающих изза случайных изъянов (засорение рогулек, изменение весового номера ровницы, нарушение вытягивающего устройства, и т.д.) и не влияющих на натяжение ровницы. Тем не менее, может иметь место несоответствие в показаниях двух датчиков, и всегда отдаётся предпочтение положению ослабленной ровницы, так как позволяет избежать опасных дефектов пряжи. Принимая во внимание, что установленное значение номинального прироста уже само по себе подходящее для правильного функционирования машины, реальный прирост будет соответствовать следующей таблице (составленной при условии номинального прироста 70 и коэффициента коррекции 10

\begin{tabular}{|c|c|c|c|c|}
\hline ССД1 & ССД2 & Выбор & Прирост & Замечания \\
\hline $\mathrm{N}$ & $\mathrm{N}$ & $\mathrm{N}$ & 70 & Нормальное функционирование \\
\hline $\mathrm{N}$ & $\mathrm{P}$ & $\mathrm{P}$ & 80 & Одна ровница натянута;целесообразно ослабить \\
\hline $\mathrm{N}$ & $\mathrm{M}$ & $\mathrm{N}$ & 70 & Нормальное функционирование \\
\hline $\mathrm{P}$ & $\mathrm{P}$ & $\mathrm{P}$ & 80 & Обе натятуты; ослабить порпоследующее вытягивание \\
\hline $\mathrm{M}$ & $\mathrm{M}$ & $\mathrm{M}$ & 60 & Обе ослаблены, сократить прирост послед.вытягивания \\
\hline $\mathrm{P}$ & $\mathrm{M}$ & $\mathrm{N}$ & 70 & Против.несоответствие,придерж.номинального прироста \\
\hline любое & $\mathrm{N}$ & $\mathrm{N}$ & 70 & Один датчик недостоверный: \\
\cline { 1 - 2 } N & любое & $\mathrm{N}$ & 70 & Придерживаться номинального прироста \\
\hline
\end{tabular}

ПРИМЕЧАНИЕ: на Стр. Дисп. 45 “Отображение формирования катушки можно ввести фиксированное изменение, равное 20 размера виртуального кулачка, для лучшей коррекции натяжения ровницы во время стадии разработки: эти изменения “вручную” введутся в действие (или прибавятся/вычтутся от значения номинального прироста) после следующего реверсирования каретки.[6]

Устранение неполадок.

Недостаточное натяжение ровницы.

- Удостоверьтесь, что номинальный прирост не очень высок.

- Если не удаётся достичь достаточного натяжения, рекомендуется снова запросить данные стрелочных датчиков о величине натянутой ровницы;

Сильное натяжение ровницы

- Удостоверьтесь, что номинальный прирост не очень низок;

- Удостоверьтесь, что значения верхних и нижних пределов не очень низкие;

- Если не удаётся достичь правильного натяжения, рекомендуется снова запросить данные стрелочных датчиков о величине натянутой ровницы; Сильное натяжение ровницы

- Удостоверьтесь, что номинальный прирост не очень низок;

- Удостоверьтесь, что значения верхних и нижних пределов не очень низкие;

Подозрение на отказ одного или более одинарных датчиков

- При зажигании на 1 сек. загораются световые индикаторы одинарных датчиков: если зажигание не полное, первый выключенный датчик может быть неисправным.

- Проверьте световые индикаторы повторителей схемы А97 и А98: если линия работает исправно, индикаторы должны мигать. 
Проверьте целостность и правильное подсоединение соединительного зажимов линии последовательной связи, которая соединяет одиночные датчики, повторители и плату ROJ A6: если соединительный зажим плохо подключен, то весь ряд датчиков не будет функционировать.

Веретена на ровничной машине размещены в два рядя в шахматном порядке, благодаря чему уменьшается площадь на одно веретено. Машина автоматически останавливается при наработке катушки заданного диаметра, при обрыве ленты, проходящей через питающее устройство, или при обрыве ровницы, выходящей из вытяжного прибора. Для быстрого останова машины на валу верхнего конического барабана установлен тормоз. Подъем и опускание нижнего конического барабанчика, а также заводка механизма управления производятся нажатием кнопок на пульте управления машиной[7].

Пыль и пух с машин удаляется пухообдувателем, который движется по рельсам, установленным над вытяжными приборами.

Принцип работы всех ровничных машин для переработки короткого волокна одинаков. Машины отличаются одна от другой устройством питающих приспособлений, конструкцией вытяжных приборов, величиной вытяжки, размерами и числом веретен (рогулек), расстоянием между веретенами, размерами паковок.

Фактическую крутку ровницы принимают значительно ниже критической, поскольку ровница на кольцевой прядильной машине подвергается вытягиванию в вытяжном приборе, а в процессе вытягивания волокна должны смещаться относительно друг друга не обрываясь. Крутку выбирают такой, чтобы ее прочность была достаточной для наматывания на катушку и чтобы ровницу можно было вытягивать в вытяжном приборе с малым усилием без повреждения волокон [8].

На ровничных машинах для кручения мычки, выходящей из вытяжного прибора, применяют веретено с рогульками. Ровница проходит в верхнее отверстие рогульки, выходит из ее бокового отверстия и затем движется внутри полой ветви рогульки; выходя из нижнего отверстия полой ветви, ровница огибает один-три раза лапку рогульки и наматывается на катушку.

На участке внутри вертикальной части полой ветви рогульки крутка ровницы меньше, чем на участке между концом полой ветви и лапкой рогульки, но больше, чем на участке внутри полой ветви, соединяющей ее вертикальную часть с головкой рогульки.

Повышенная крутка ровницы на этом участке объясняется распространением части ложной крутки с участка между концом полой ветви и лапкой рогульки, а также образованием дополнительных витков крутки при взаимодействии ровницы с концом полой ветви. (Сказанное относится к обычной рогульке.) Используя рогульки эффективной крутки, можно значительно увеличить крутку ровницы и ее прочность внутри вертикальной части полой ветви.

Каждый последующий слой ровницы на катушке уменьшается по высоте на постоянную величину. Благодаря этому катушка ровницы получает форму цилиндра, ограниченного сверху и снизу конусами.

\section{Список использованной литературы}

1. Джуманиязов К.Дж., Гофуров К.Г., Матисмаилов С.Л. и голова. Технология и оборудование текстильных изделий. Учебник. - Т.: Г.Гулом, 2012. Стр. 132.

2. Столяров А.А. и др. Прядение натуральных и химических волокон. Текст лекций. Иваново. 2015. 15 ст.

3. Разумеев К.Э. и др. Теоретические основы технологии прядения. Иваново. 2019. 78 ст.

4. Kazakov F.F. Сатторова. H.H. International Journal of Advanced Research in Science, Engineering and Technology ISSN: 2350-0328 Vol. 6, Issue 11, November India 2019. p. 11658-11663

5. Саидов Б. Ю, Казаков Ф.Ф., Саторова Н.Н. Научно-технический журнал” развитие науки и техники". №1.Бухара 2020

6. S.Kh.Fayziyev, Kh.K.Rakhimov. Creation of a New Design of a System for Feeding raw Cotton to a Cylinder, International Journal of Recent Technology and Engineering (IJRTE) ISSN: 2277-3878, Volume-8, Issue-4, November 2019. p.12753-12759

7. Kh.K.Rakhmonov, S.Kh.Fayziyev. Study of Effect of Speed and Temperature of the Drying Agent in the FeederLoosened of New Design on the Quality of Fiber. International Journal of Emerging Trends in Engineering Research, Volume 8. No. 10, October 2020, p. 7008-7013

8. Kazakov F.F. Kodirov T.J. Tanning of astrakhan skins modified by carboamid of ormaldhyde resin. European science review.№ 7-8 (Jul-August). ISSN 2310-5577. Vienna-Prague 2017. p.100-103 